International Journal of Solar Thermal

Vacuum Engineering

\title{
Experimental and theoretical performance evaluation of parabolic trough mirror as solar thermal concentrator to thermoelectric generators
}

\author{
Saim Memon ${ }^{1,}{ }^{*}$, Maekele Mihreteab ${ }^{1}$, Takao Katsura2 ${ }^{2}$, Ali Radwan ${ }^{2,3}$, Shanwen Zhang ${ }^{4}$, Ahmed A \\ Serageldin ${ }^{2,3}$, Essam M. Abo-Zahhad ${ }^{5}$ \\ 1 Solar Thermal Vacuum Engineering Research Group, London Centre for Energy Engineering, School of Engineering, London South Bank \\ University, 103 Borough Road, SE1 OAA, London, UK \\ 2 Division of Human Environmental Systems, Faculty of Engineering, Hokkaido University, Sapporo, Japan \\ 3 Mechanical Power Engineering Department, Faculty of Engineering, Mansoura University, El Mansoura 35516, Egypt \\ 4 College of Mechanical Engineering, Yangzhou University, Yangzhou 225127, China \\ 5 Mechanical Power Engineering Department, Faculty of Energy Engineering, Aswan University, Aswan 81528, Egypt
}

\section{ABSTRACT}

This paper presents the prospects of harnessing radiative heat from the sun with a parabolic trough mirror, as a solar thermal concentrator, in comparison to the mathematical model and experimental quartz-halogen concentrator model for the electrical energy conversion utilizing thermoelectric generators (TEG). The construction and design of TEG-setup along with Parabolic trough mirrors and quartz-halogen lamps are presented. The Parabolic trough mirror used as a focal point at a distance of $19.05 \mathrm{~cm}$. With eight quartz-halogen concentrated heat, the maximum performance achieved at $\Delta \mathrm{T}$ of $11.8 \mathrm{~K}, \mathrm{~V}_{\mathrm{oc}}$ of $292 \mathrm{mV}$ and $\mathrm{I}_{\mathrm{sc}}$ of $95.8 \mathrm{~mA}$, recorded at the concentrated hot-side surface temperature of $317.8 \mathrm{~K}$. When compared to the natural solar concentrated heat, higher temperature of $473.15 \mathrm{~K}$ at the hot-side surface temperature of TG was achieved. It is concluded that the heat concentration of the parabolic mirror increases with an increase in the intensity of heat using natural solar radiations. The $\mathrm{V}_{\text {oc }}$ of $1.76 \mathrm{~V}$ and $\mathrm{I}_{\mathrm{sc}}$ of $1.1 \mathrm{~A}$ at a temperature difference of $110 \mathrm{~K}$ were measured, which are in good agreement with validated mathematical results. The parabolic through mirror utilized is smaller in size and thus collected lesser sun rays than the larger dish style mirror, and hence the heat in the focal point was very low, for better results, parabolic trough mirror with higher surface area would be important for future experiments.

Keywords:

Solar Thermal Energy; Thermoelectrics; Waste Heat Recovery; Parabolic Trough Mirror; Electrical Performance

Copyright @ 2020 PENERBIT AKADEMIA BARU - All rights reserved

\section{Introduction}

Due to an aggregating consternation of carbon emissions and need of harmonizing energy supplydemand gap, harnessing unused radiative heat from the sun and its conversion to electrical energy with thermoelectric generators (TEG) have enlightened the attentions to curb climate change [1]. The application of TEG is also more associated with the conversion of waste heat from electric or gasoline vehicles and glass/steel/mechanical instruments production factories [2,3]. A recent study

\footnotetext{
* Corresponding author.

E-mail address: S.Memon@lsbu.ac.uk
}

https://doi.org/10.37934/stve.1.1.2238 
shows the conversion of waste heat from domestic boilers to electrical energy has also opened up the scope of thermal energy management for prospective zero energy buildings [4]. TEG can be used in small applications from lesser power demand areas to much higher power generation but it comes with the repercussions of lower conversion efficiency due to the limitations of thermoelectric materials [5]. Traditionally, thermoelectric materials consist of semiconducting material of two different metals of $\mathrm{p}$-type and n-type materials. This $\mathrm{p}$-type and $\mathrm{n}$-type materials are known for transporting electrons from the hot side of the material to the cold side and vice versa. The Seebeck effect occurs when two metals of different material properties and at different temperatures are connected electrically in series and parallel in connection, an electrical voltage is generated across the two dissimilar junctions of the material $[6,7]$. In order, the TEG to have higher efficiency, the $p$ type and n-type material should have the same length and same cross-sectional area but with a higher figure of merit ZT and the temperature difference between cold and hot side [8]. The hot side hypnotizes heat from the source of energy; then the heat starts to descend in exits past the cold material when heat passes through the cold side positive electrons from the $p$-type and negative from the n-type electrons flows to the load. The concept of TEG was discovered in 1822 by Johan Seebeck when deflection of the needle on the electrical apparatus occurred due to the dissimilar metals at dissimilar temperatures. This result due to the temperature change upon the junction of two semiconducting metals of different temperatures [9]. After Seebeck discovery, the research upon this material increased. Then Oersted discovered the communication between the electric current and a magnetic needle.

Many famous scientists did research for understanding the relationship between electric current and magnetic field. Then the scientists able to understand that the Seebeck opinion was not occurred because of the magnetic polarization it is caused but because of the current flowing in the closedloop system [10]. Seebeck coefficient is related with a couple designed by two materials; later, the Thomson effect revealed that a total Seebeck coefficient could be associated with each material individually. Thermoelectric power is generated from the cold side to the hot side, in which positive p-type electrons flow from the hot side to the cold side for the negative for $n$-type conduction. In the year 1834 watchmaker and scientist Jean Peltier informed temperature variance at the junction of two dissimilar material as current was passed through the junction [11]. In 1838, Lenz cooled water and freeze and melted it by changing the direction of the current. In this way, Lenz introduced the first thermoelectric cooler.

About $63 \%$ of fuel energy wasted as a waste heat [3]. For reusing the wasted heat, an improvement to thermoelectric material is essential. Whilst, semiconductors are found to have the highest thermoelectric material with carrier concentration about $10^{22} \mathrm{~cm}^{-3}[5]$. There are several factors affecting the performance of the thermoelectric generator. For the best thermoelectric material, there are three factors to be considered for improvement of its performance: higher temperature difference, higher electrical conductivity, and lower thermal conductivity. Additional performance issues that can be considered for achieving maximum power is that the material should be made from thermoelectric material with different signs: $p$-type material for the positive side and n-type material for the negative [13]. Conventional thermoelectric materials are $\mathrm{Bi}_{2} \mathrm{Te}_{3}, \mathrm{PbTe}, \mathrm{Si}_{1-x}$ $\mathrm{Ge}_{x}$, and $\mathrm{N}$-type $\mathrm{Bi} \mathrm{Sb}, \mathrm{Bi}_{2} \mathrm{Te}_{3}$ shows the highest performance near room temperature [14]. A good positive thermoelectric material $Q>$ zero is a solid solution of composition $25 \%: 75 \% \mathrm{Bi}_{2} \mathrm{Te}_{3}: \mathrm{Sb}_{2} \mathrm{Te}_{3}$. For designing the best negative $n$-type thermoelectric material, the value of $Q$ should be less than zero $(\mathrm{Q}<0) 25 \%: 75 \% \mathrm{Bi}_{2} \mathrm{Te}_{3}: \mathrm{Bi}_{2} \mathrm{Se}_{3}$ is also a solid solution [7]. The demand for thermoelectric material is high: They can be used as coolers, excess temperature regaining in many industries and vehicles, sensors, and detectors power generation. They have several advantages to fuel generators: they have 
excellent duration, no moving parts, silent operation, and no chemical refrigerating parts. As mentioned above, there are several factors, which affect the performance of TEG.

The substantial parameters for improving the conversion efficiency performance are not only about developing advanced thermoelectric materials but more of utilizing the radiative heat; in this case concentrated solar power to TEG but it is also important to discuss the significance of Seebeck effect, Peltier effect, Thomson effect and figure-of-merit ZT.

\subsection{Seebeck Effect}

Seebeck effect is one of the thermoelectric effects that has a great influence on the discovery and performance of TEG. The TEG is formed of two different metals of different temperature and material properties [15]. From construction of thermoelectric generator most of the time it contains two surface which are exposed to different temperature, the hot side temperature $\left(T_{H}\right)$ and to the cold side temperature $\left(T_{C}\right)$ and are connected by thermoelectric couples or legs ( $P$ type and $n$ type) materials. When the $T_{H}$ is greater than the cold side temperature $\left(T_{C}\right)$ an open circuit electromotive force (emf) can develop between the two junctions [4]. The output potential difference or voltage is the result of the Seebeck coefficient and temperature difference between the hot side and cold side of the TEG $[15,16]$. The Seebeck effect is not affected by factors such as change in cross sectional area, temperature sharing and its temperature gradient but it is the function of temperature difference between the junctions of dissimilar materials [3].

\subsection{Peltier Effect}

Peltier effect is described as the reverse process of the Seebeck effect. Peltier effect current is applied to the circuit from an external heat source. Because of this, the rate of heat flow $q$ appears at one junction and $-q$ at another junction [3]. This process is mostly applied in refrigerators as a cooling system to absorb heat from an object and creates cold atmosphere. The absorption and liberation of heat related to the Seebeck coefficient, when the applied electric current and Seebeck current are in the same direction then heat is absorbed in the hotter junction and heat liberates at the cold junction [17]. Most of the time the flow of current determines the liberation and absorption of heat and it does not affected by factors such as shape and dimension of the material forming the junction, but it is the result of temperature of the junction and configuration of the material. The Peltier effect formula then given by $\pi=\frac{I}{q}[16,15]$.

\subsection{Thomson Effect}

Thomson effect is one of the thermoelectric effects, which has lower effect on its performance, but its effect depends on the value of $\Delta T$. Thomson effect defined as generation of heat when current passes along a slice of a single conductor, which has a temperature difference $\Delta T$. When the temperature difference is smaller the Seebeck coefficient is smaller $[8,16]$. When $1 \mathrm{~K}$ of temperature gradient passes through a conductor heat content of a single conductor changes per cross sectional area of that conductor it is known as Thomson effect $[17,18]$.

\subsection{The figure-of-merit $Z T$}

The dimensionless figure of merit ZT has great influence on the performance of thermoelectric generator [15]. Increasing the value of the figure of merit ZT is dependent on the material properties 
of the material used for construction of thermoelectric module $[6,11] . \mathrm{Bi}_{2} \mathrm{Te}_{3}$ is prominent composite, which has great values of figure merit $Z T$ and is the best option of the manufacturers due to handling of the greater temperature saturation points. Enhancing the value of ZT indicates the conversion efficiency of the TEG is high which leads to high output power $[19,20]$.

\subsection{Parabolic trough mirror as a solar thermal concentrator to thermoelectric generator}

The research results presented in this paper, for the first time, with the concentrated heat energy harvesting scope correlated to parabolic trough mirror in improving the temperature difference of the TEG, a schematic diagram is shown in Fig. 1. The parabolic trough mirror works in several ways, the main function is to collect solar rays from a larger surface area and to concentrate it into smaller surface area. For better performance and for effective output it is essential to find the focal length of the parabolic through mirror. The focal point is the point where all rays meet, and the highest temperature is created at that point. The focal point of the parabolic trough mirror is some distance away from the surface of the mirror and at the centre of the diameter of the mirror. Eq. (1) shows the method to calculate focal length [21]. Focal length, as shown in Fig. 1, is represented by letter $f$ and depth of the mirror is represented by small $d, D$ is diameter of the mirror. The focal length of the parabolic trough mirror used in this experiment is $19.05 \mathrm{~cm}$ and diameter of $15.2 \mathrm{~cm}$ with a depth of $0.74 \mathrm{~cm}$.

$f=D^{2} / 16 d$

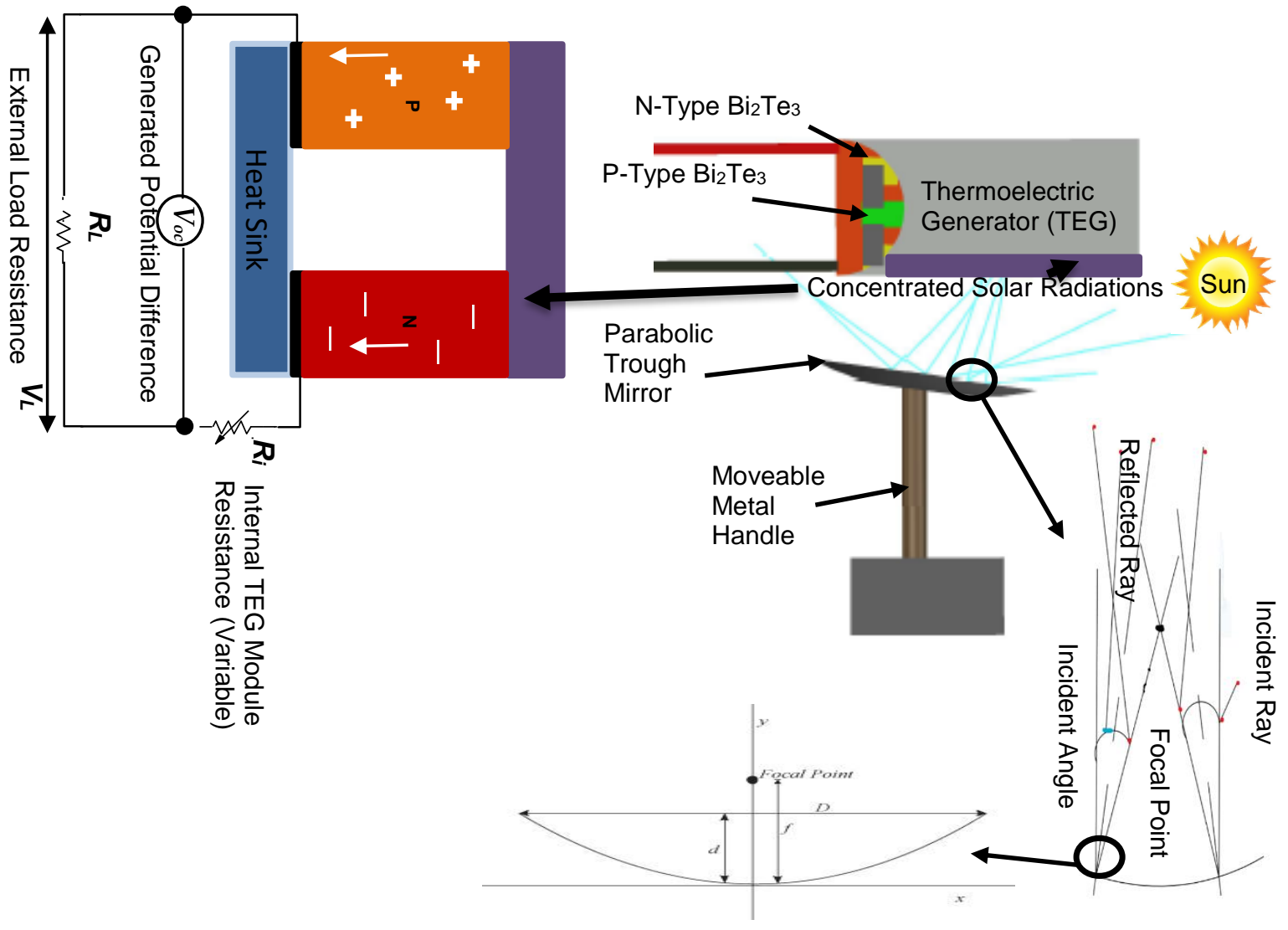

Fig. 1. Schematic diagram of parabolic trough mirror as a solar thermal concentrator to Thermoelectric Generator (TEG) consists of $\mathrm{N}$ and $\mathrm{P}$ and type $\mathrm{Bi}_{2} \mathrm{Te}_{3}$ 
As shown in Fig. 1, when an incident rays falls on a parabolic mirror the reflected rays are converged into the focal point of the mirror. From this, all reflected rays pass through the focal point and concentrates on its focal point where the highest temperature collected by the mirror to be applied on the hot side of the TEG. As the focal point is on the focal axis of the mirror all the parallel rays reaching the surface of the mirror reflected and passes through the focal point. The angle of incidence is equal to the angle of reflection. Fig. 1 shows the depiction of incident ray, reflected ray and normal ray. It shows the incident ray and reflected ray passing all reflected rays through the focal point and plotting normal ray in between the rays, the same angle of incident and angle of reflection $9^{\circ}$ was achieved.

This study experimentally evaluates the influence of higher concentrated temperatures on the open circuit generated voltage compared to the standard performance parameters. The research results presented provide practical discernment into the testing (open-short-full load) of TEG in understanding the performance limitations of it. The analytical simulation and mathematical model developed in MATLAB compared to the electrical performance parameters and its dependencies. The measurements and values of the parameters used are obtained from experiments to test and understand the comportment of TEG modules as it is essential for defining the performance of TEG. The output of the experiments are clearly discussed and plotted, for better understanding of its behavior, for defining the theoretical explanation of the TEG. Plots of conversion efficiencies, temperature difference, open circuit voltage, short circuit current and dimension-less figure-of-merit ZT are included in the results. A number of experiments were conducted but reported in this study two that are: the TEG-setup performance test using eight quartz-halogen lamps as heat concentrator with parabolic trough mirror and TEG-setup with natural sunlight as solar thermal concentrator with parabolic trough mirror. The results calculated and obtained from the experiments are open circuit test, short circuit test, load power test, hot side temperature and cold side temperature. The other parameters, which influence the performance of TEG, are calculated using MATLAB and the theoretical results are in good agreement with the experimental results.

\section{Mathematical Modelling Approach}

The mathematical model of the TEG is validated and established in MATLAB that work on the principle of effects mentioned above and the output predictions of electric potential under the influence of the temperature difference of cold and concentrated solar-heated hot side [22]. The larger the temperature difference, the greater the out electrical power. However, the temperature difference is not easy to obtain, due to which an effective cooling mechanism was required to keep one side cooler than the other [23]. As the large temperature difference is difficult to obtain, it means that the overall efficiency of the TEG is very low. An increase in the overall efficiency is primarily dependent on thermoelectric materials which play a significant part in determining its operating temperature range, and their range is usually divided into three categories: less than $450 \mathrm{~K}$ utilize Bismuth (Bi) composite with Tellurium (Te), Antimony (Sb), and/or Selenium (Se) basis of commercial TEGs; 600K-850K utilize composites of Lead (Pb) and Telluride (Te); and 900-1300 K utilize Germanium ( $\mathrm{Ge}$ ) and Silicon (Si). Thus, $\mathrm{Bi}_{2} \mathrm{Te}_{3}$ based TEG is chosen due to its reasonable operating temperature range of $<450 \mathrm{~K}$. They are usually coupled in series and packed between heatconductive elements providing heat from the concentrated solar heated source and cooling from the heat sink to achieve a reasonable power. When temperature gradient occurred between $\mathrm{P}$ and $\mathrm{N}$ junctions of $\mathrm{Bi}_{2} \mathrm{Te}_{3} \mathrm{TEG}$ it is found that, the generated potential difference or voltage $\mathrm{V}$ shows up on the voltmeter and is represented in Eq. (2) [25]. 
$V_{o c}=\alpha\left(T_{h}-T_{c}\right)$

where $V_{o c}$ is the open-circuit voltage, $\alpha$ is the Seebeck coefficient, $T_{h}$ is the concentrated solar heat hot-side temperature, and $T_{c}$ is the cold side temperature enhanced further with ice-cubes. The $\alpha$ can be achieved by using Eq. (3).

$\alpha=\frac{V}{\Delta T}$

Equation (3) shows the Seebeck coefficient is inversely proportional to the temperature difference $\Delta T$ between the hot side and cold side of TEG. For better results and for increasing the Seebeck coefficient the temperature of the cold side has kept in a low temperature. The electrical current of the TEG was calculated using Eq. (4).

$I=\frac{2 N \alpha\left(T_{h}-T c\right)}{R_{L}+R e}$

The TEG electrical power generation at the load of the circuit is then calculated by using Eq. (5).

$P=I^{2} * R_{L}$

The hot side heat flow from the concentrated solar heat through parabolic trough mirror is found by Eq. (6).

$Q_{h}=n\left[\alpha * T_{h} * I-\frac{1}{2} * R e * I^{2}+K\left(T_{h}-T_{c}\right)\right]$

The cold side heat flow of the TEG was obtained by Eq. (7).

$Q_{c}=n\left[\alpha * T_{c} I+\frac{1}{2} * R e * I^{2}+K\left(T_{h}-T_{C}\right)\right][16]$

Some of the parameters that affects the conversion efficiency of TEG are temperature dependent figure of merit $Z T$ consists of figure of merit of $Z$, thermal conductivity, electrical conductivity, electrical resistivity and absolute temperature and are paramount of determining the maximum efficiency using Eq. (8). [26]

$\eta_{\text {max }}=\left(1-\frac{T_{c}}{T_{h}}\right) \frac{\sqrt{1+Z T}-1}{\sqrt{1+Z T}+\frac{T_{c}}{T_{h}}}$

The dimension less temperature dependent figure of merit $Z T$ value which has great influence in conversion efficiency of TEG obtained by using Eq. (9).[26, 27].

$Z T=\frac{\alpha^{2} \sigma T}{K}$

The temperature independent figure of merit $Z$ is obtained by $[28,29]$.

$Z=\frac{\sigma \alpha^{2}}{K}$ 
The value of figure merit $Z T$ is dependent of the average temperature $T_{A V}$ and is gained by the following formula.

$T_{A v}=\frac{T_{h}+T_{C}}{2}$

The thermal conductivity of the TEG was calculated using Eq. (12) [15]

$K=\frac{Q L}{\Delta T A}$

The electrical resistivity is given by Eq. (13).

$\rho=\frac{R A}{L}$

The electrical conductivity is given by Eq. (14).

$\sigma=\frac{1}{\rho}$

The short-circuit current determining the maximum current the TEG could draw is given by Eq. (15) $[8,9,13,18]$.

$I_{S C}=\frac{2 \alpha T_{a v}\left(1-\frac{T_{c}}{T_{h}}\right)}{R_{e}\left(1+\frac{T_{C}}{T_{h}}\right)}$

The open circuit voltage of TEG for both P-type and N-type material given by Eq. (16). [30]

$V_{o c}=\frac{2 n \alpha T_{a v}\left(1-\frac{T_{c}}{T_{h}}\right)}{\left(1+\frac{T_{C}}{T_{h}}\right)}$

The maximum power of TEG is achieved when the load resistor is equivalent to the internal resistance of the TEG $\left(R_{L}=R_{e}\right)$. Maximum power of TEG given by Eq.(17). [28]

$P_{\max }=I_{m p}^{2} * R_{L}, P \max =\left[2 N \alpha\left(T_{H}-T_{C}\right)\right]^{2} / 4 R_{L}$

The maximum power efficiency is given by Eq. (18). [30]

$\eta_{m p}=\frac{\left(1-\frac{T_{c}}{T_{h}}\right)}{2-\frac{1}{2}\left(1-\frac{T_{c}}{T_{h}}\right)+\frac{2\left(1+\frac{T_{c}}{T_{h}}\right)}{Z T}}$

Voltage at maximum power point is given by Eq. (19).

$V_{m p}=\frac{n \alpha\left(T_{h}-T_{C}\right)}{2}$

Electrical current at maximum power point is given by Eq.(20). [11,30] 


$$
I_{m p}=\frac{\alpha\left(T_{h}-T_{C}\right)}{2 R}
$$

\section{Experimental Setups and Methods}

In these experiments, TEG (SP1848-27145), size of $40 \mathrm{~mm} \cdot 40 \mathrm{~mm} \cdot 3.4 \mathrm{~mm}$, both P and N type elements $\left(\mathrm{Bi}_{2} \mathrm{Te}_{3}\right)$ insulated with Teflon connected with copper lead, were used. Several experiments were conducted in achieving the appropriate mechanism in harnessing the concentrated radiations to electrical power conversion. A flat metal, connected to the TEG acted as a hot side surface, polished with sand paper and coated with a heat resistant $\left(220^{\circ} \mathrm{C}\right)$ black colour paint to protect the TEG from the thermal damage when heat is directly applied on the hot side of TEG and for minimizing loss of heat by reflection. Initial, testing was conducted with hot-plate with external heat sink to keep the cold-side temperature less than $308 \mathrm{~K}$, supported with small cooling fan, and the heat-source (hot-plate) temperature goes around $419 \mathrm{~K}$ and was subjected to a range of thermo-electrical conditions for the electrical performance testing. The open-circuit voltage $\left(\mathrm{V}_{\text {oc }}\right)$ test was conducted when the circuit is not connected with external load resistor $\left(\mathrm{R}_{\mathrm{L}}\right)$. The short-circuit current test was conducted when the output leads are shorted with copper conductor. The full-load test was conducted when the TEG leads are connected to external load resistor $\left(R_{L}\right)$ and for maximum power measurement when $R_{L}=R_{e}$, for this reason a variable resistor of $100 \Omega$ was utilised to measure the maximum power performance. In these initial tests, the hot and cold side of TEG was analysed by connected it into the digital multi meter to the input leads of TEG. Negative voltage reading indicated the cold side and positive voltage for the hot side. In this way, the hot side and cold side of TEG are determined. Once the sides are identified, the TEG is bolted on the black coated surface using thermal glue, facing the hot side to the surface, which is ready to be exposed to the concentrated heat. On the cold side of TEG heat sink and fan is connected to keep cool the cold side and the TEG is ready for experiment.

\subsection{TEG-setup with quartz-halogen radiative concentrated heat testing components}

The parabolic trough mirror, diameter of $15.2 \mathrm{~cm}$, having the distance of focal point from centre positioned to be $19.05 \mathrm{~cm}$ at depth of $0.74 \mathrm{~cm}$. The multi-logger temperature recorders employed for the measurement of both hot side and cold side temperatures whilst the mirror is set on top of the TEG. The digital multi meters consists of power analyser, voltmeter, ammeter and accuracy checks were conducted with balanced digital multi meter were connected to the TEG-setup. The TEG-setup consists of heat sink on the cold-side immersed in the ice-cubed container and the hot-side positioned to parabolic trough mirror. Whilst, in this part of the experiment eight quartz halogen lamps, each $500 \mathrm{~W}$, were utilised in the controlled laboratory environment. The number of quartzhalogen lamps increased to eight because it was not suitable to focus all lamps in a single working area as it has hinged on a metal plate in a space of $25 \mathrm{~cm}$ apart from each other on two metal roads. The heat on the surface area and focal point achieved with 8 quartz-halogen lamps were better than the initial 2 to 4 quartz-halogen lamps. The purpose of these quartz-halogen based experiments was to test the behaviour of TEG by concentrating heat on the hot side of TEG achieving the right focal point of concentration, which was generated by the eight halogen lamps, using parabolic trough mirror as heat concentrator. By connecting all the experimental equipment, open voltage measurement and short circuit measurements were recorded. Fig. 2 shows the TEG-setup with eight quartz-halogen lamps sufficient to emit the solar radiations to be harnessed as part of initial tests. 

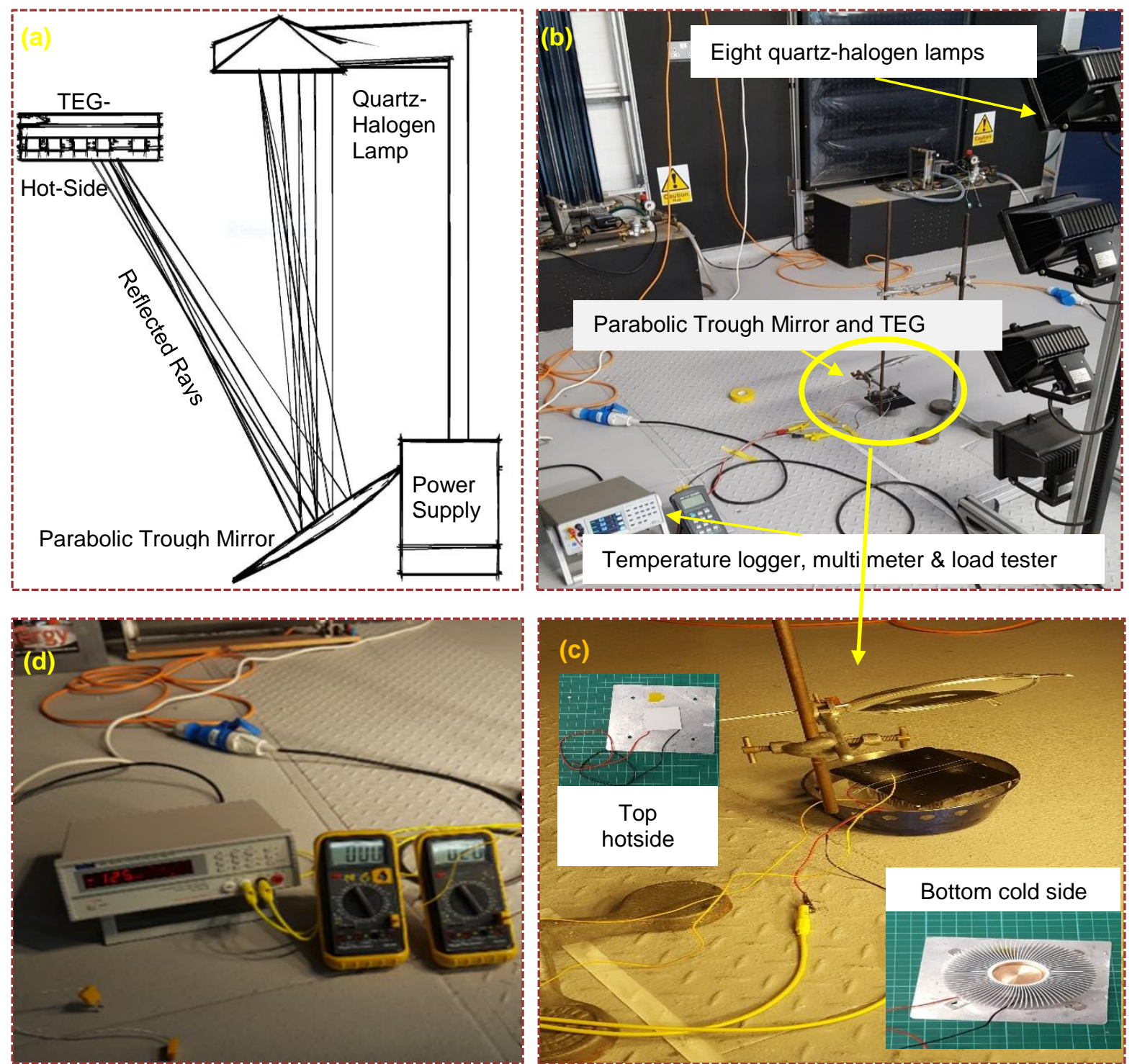

Fig. 2. Experimental TEG-setup with quartz halogen radiative concentrated heat testing components showing (a) projected sketch matching the focal length, (b) four quartz halogen lamps positioning in achieving concentrated temperatures (c) on the hot side of the TEG whilst the cold-side submersed with ice-cubed container and (d) connected to live measurement digital meters

\subsection{TEG-Setup with Natural Solar Thermal Concentrated Heat Testing Components}

In this part of the experiments, natural sun light, at 13:00 GMT in London, was utilised to concentrate the solar radiations at focal point of the hot-side of TEG-setup. Surface temperatures raised as expected and the results were recorded. Focusing of the mirror and cooling the cold side has been challenging. The cooling technique utilised achieved the reduction of cold side temperature of TEG setup. Fig. 3 shows the complete experimental setup and the required equipment for measuring open circuit voltage and short circuit current and surface temperatures with k-type thermocouples. Two multi meters were connected to the cold side and hot side of the metal plate using k-type thermocouples for measuring the surface temperature on the cold side and hot side of TEG. Focusing the mirror and finding the right focal point was challenging because the focal point of the mirror is at the centre of its diameter and in front of the mirror. The focal point of the mirror was not suited for cooling technique used. The focusing technique used in both sunlight and halogen 
lamps were different. In the halogen lamp experiment, focusing the mirror down to the TEG brought better result than putting it parallel with the TEG. It is because of the connection of lamps, the lamps were hinged to a metal, which is not suitable for repositioning. In sunlight experiments, focusing the mirror downward to the TEG achieved maximum concentrated surface temperature of $193^{\circ} \mathrm{C}$ in coldarid London, England, climate. The cooling technique used in this experiment can be improved with micro-channel-based heat-sink with better cooling mechanism, but the current technique reduced the cold side temperature. A metal plate was inserted in between the ice and the heat sink to avoid direct contact of ice with the heat sink. Other benefits of using the metal plate was for been sure no water droplets reach the TEG. The bowl which is holding the ice has holes on its top the reason of choosing this kind of container is the melted ice to leave the bowl as a water without causing any damage to the TEG.

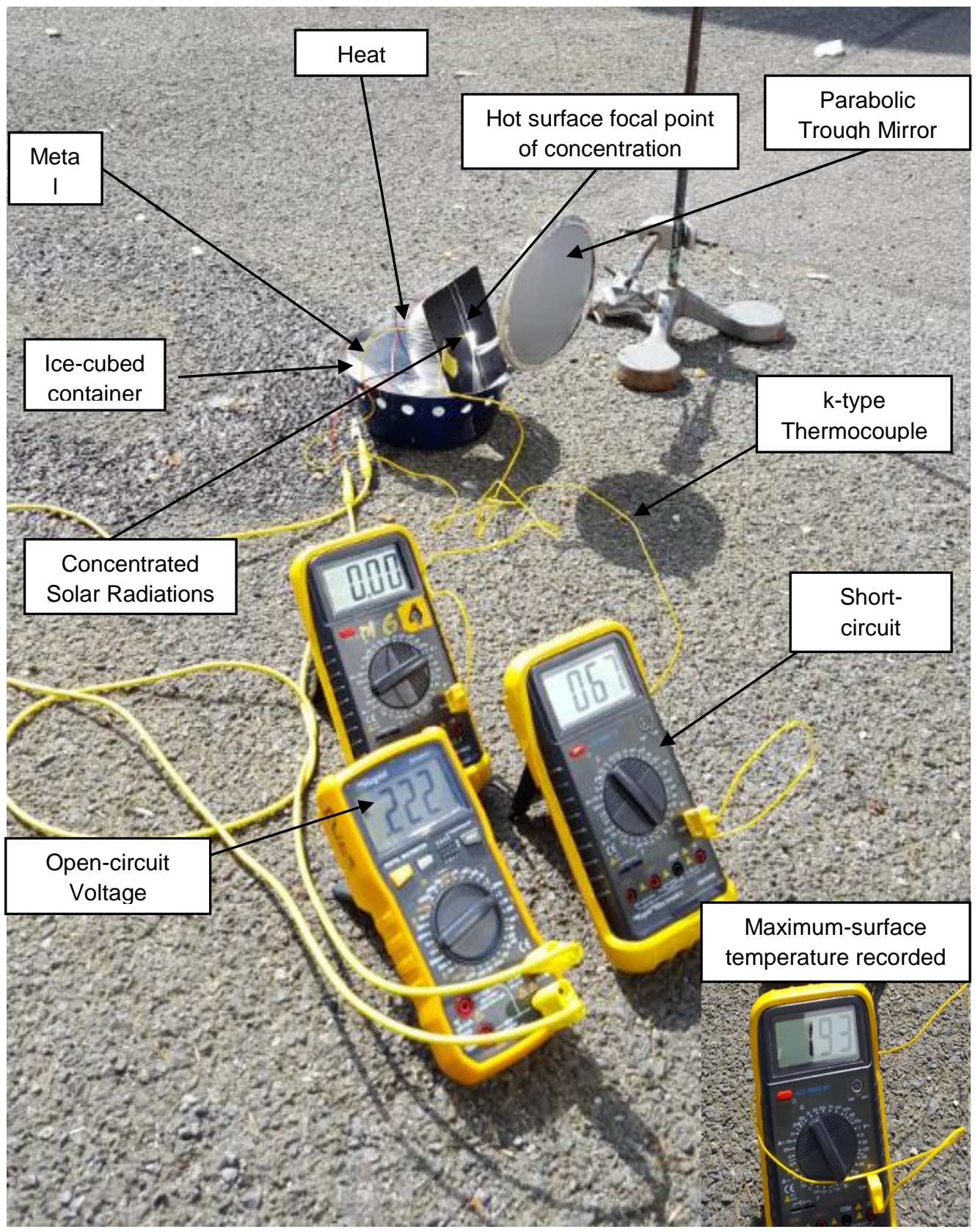

Fig. 2. Experimental TEG-setup with natural solar thermal concentrated heat testing components 


\section{Results and Discussions}

\subsection{Mathematical results of maximum power point full load analysis at dynamic temperatures}

Figure 3 predicts the performance of TEG-setup at maximum power point effect of full load tests at four different $\Delta T$ on the output load power $(P)$ connected to the variable load resistor $\left(R_{L}\right)$ when $\mathrm{R}_{\mathrm{L}}=\mathrm{R}_{\mathrm{e}}$. It can be seen that with an increase of $\Delta T$ increases the maximum power-point, which validated the model predictions reported elsewhere $[3,25]$. The maximum power point occurs when $V_{L}$ and $I_{L}$ are approximately equivalent to the half of the $V_{o c}$ and $I_{s c}$, respectively. However, the $R_{e}$ is dependent on $\Delta T$ and is variable. The output load power $\left(\mathrm{P}_{\mathrm{L}}\right)$ at maximum power point of $0.44 \mathrm{~W}$ occurred at approximately $I_{L}$ of $0.32 \mathrm{~A}$ at $\Delta T$ was continued at $60 \mathrm{~K}$ due to less direct-heat supply causing a decrease in current and $R_{L}$ in this case when it is under-loaded.

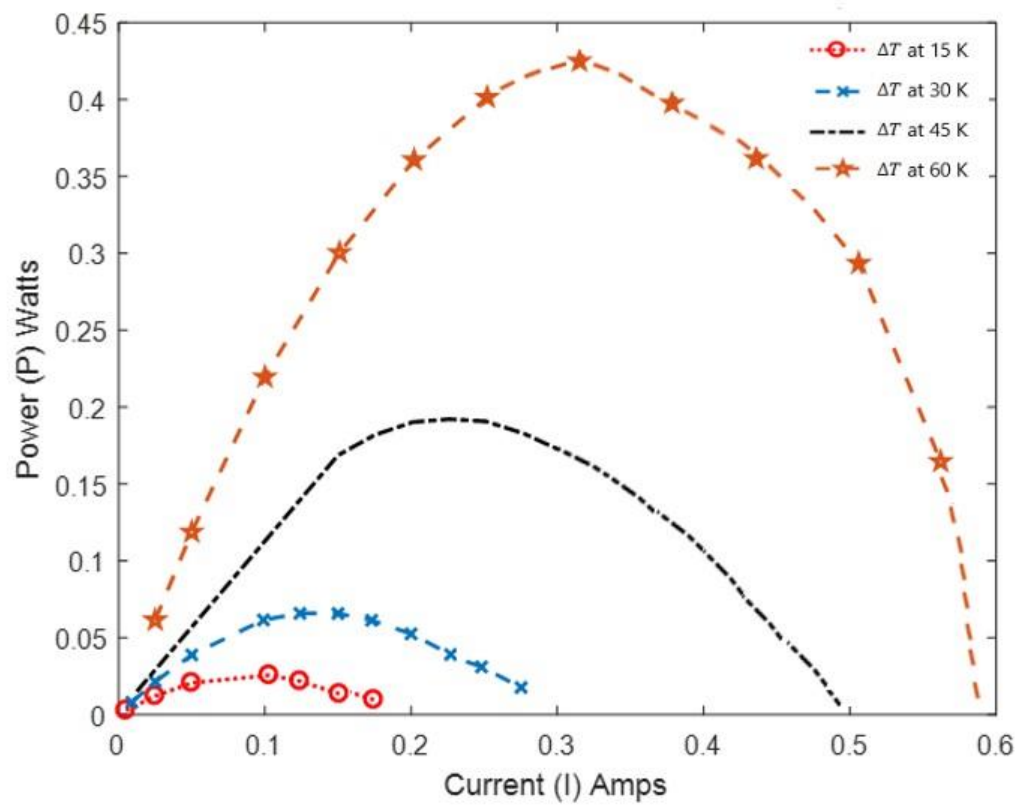

Fig. 3. Mathematical analysis of the maximum power point effect of full load tests at four different $\Delta T$ on the output load power $(P)$ connected to the variable load resistor $\left(R_{L}\right)$ when $R_{L}=R_{e}$

\subsection{Experimental Results of TEG with Quartz-Halogen Radiative Concentrated Heat}

The use of eight quartz-halogen radiative concentrated heat experiments are sensitive and must comply with health and safety regulations. For this, the minimum distance of $1 \mathrm{~m}$ was kept between the TEG-setup and the hot-surface of the quartz-halogen lamps so that the intensity of heat that reached the surface area of the parabolic trough mirror along the safe measurements of $V_{o c}$ and $I_{s c}$. Figure 4 shows the main contributor in enhancing the $V_{o c}$ and $I_{s c}$ is the results of the temperature difference between the hot and cold sides. As temperature-difference rises in the contact area the output voltage and current of TEG increases. However, due to the concentrated temperature rise on the hot side of TEG reduced the temperature difference. This was improved by cooling the cold side of TEG with ice-cubed container. A higher the temperature difference the better result were measured. At $\Delta T$ of $11.8 \mathrm{~K}$, higher $V_{o c}$ of $292 \mathrm{mV}$ and $I_{s c}$ of $95.8 \mathrm{~mA}$ and was recorded at the concentrated hot-side surface temperature of $317.8 \mathrm{~K}$. The limitation of these results are that the full potential of $\Delta T$ could not be experimentally possible under this setup. However, the results are in good agreement with the validated mathematical model of the TEG setup. These results are validated with similar thermoelectric behaviours reported elsewhere [11, 22]. 
Figure 4a shows the relationship between the differential temperature, $\Delta T$, against the opencircuit voltage, $V_{o c}$. The values of $\Delta T$ is measured using the k-type thermo-couple and the output values such as short-circuit current, $I_{s c}$, and $V_{o c}$ are measured using digital multi meter. The change in the temperature difference has significant influence on the output values. When $\Delta T$ increases the $V_{o c}$ increases and $I_{s c}$ also increases, as shown in Fig. 4b. In this experiment, a maximum of $11.8 \mathrm{~K} \Delta T$ is measured giving a maximum of $I_{s c}$ of $0.096 \mathrm{~A}$ and maximum $V_{o c}$ of $0.295 \mathrm{~V}$. From this by increasing $\Delta T$ of the TEG maximum output values can be achieved. Fig. $4 \mathrm{c}$ and $4 \mathrm{~d}$ shows output power measurements and calculated $Z T$ parameters against the differential temperature. The with an increase of temperature dependent figure of merit the output power can be increased and thus dependent on the TEG constructional materials. However, the output power is insufficient due to the hot side temperature measured from the increased halogen lamps used was very low even at the right calculated focal point. At a maximum temperature difference, $\Delta T$, of $11 \mathrm{~K}$, maximum output power of 0.04 is measured. Fig. $4 \mathrm{e}$ and Fig. $4 \mathrm{f}$ shows the $V_{o c}$ and $I_{s c}$ relationship against the average temperature, respectively. The $V_{o c}$ and $I_{s c}$ increases linearly with respect to the change in average temperature difference. It illustrates that at $480 \mathrm{~K}$ of avarage temperature, $T_{a v}$, maximum $I_{s c}$ and $V_{o c}$ of $0.1 \mathrm{~A}$ and $0.3 \mathrm{~V}$, repsectively, are measured. However, the graphs of $\Delta T$ versus $V_{o c}, I_{s c}$ and $T_{a v}$ shows similar behaviour because the main influence on the outputs of TEG is indipendent of avarage temperature or other factors but is results of $\Delta T$ of the TEG. Fig. $4 \mathrm{~g}$ shows the relationship between the $T_{a v}$ and the temperature dependent figure of merit $Z T$. It shows that the avarge temperature has great influence on $Z T$.

\subsection{Experimental Results of TEG with Natural Solar Thermal Concentrated Heat}

The experimental results obtained using natural sunlight, as source of solar thermal concentrated heat considered to be better than the experimental results obtained using quartz-halogen heat. This is because of the intensity of sunlight is stronger than the quartz-halogen lamps and therefore the parabolic trough mirror concentrated up to $473.15 \mathrm{~K}$ or $200^{\circ} \mathrm{C}$ as shown in Fig. 5. Measurements show an increase of short circuit current and open circuit voltages. The surface temperature raises from $295.3 \mathrm{~K}$ to its maximum $466.3 \mathrm{~K}$. After this, the concentrated heat on the surface gradually decreased to $372.15 \mathrm{~K}$. This is due to the change in the direction of the solar irradiance and azimuth angle was needed to be refocused with focal point aligned for harnessing the maximum possible concentration of heat. By tracking the sun and moving, the mirror into the direction where the sun moves the maximum temperature was regained. The experimental results are in good agreement with [31]. The $V_{o c}$ of $1.76 \mathrm{~V}$ and $I_{s c}$ of $1.1 \mathrm{~A}$ at a temperature difference of $110 \mathrm{~K}$ were measured, this is in good agreement with the results of [32].

Figure 5a shows the nonlinear relationship of $V_{o c}$ against $\Delta T$, as the maximum $V_{o c}$ of $3.35 \mathrm{~V}$ achieved at $\Delta T$ of $110 \mathrm{~K}$. After its peak, due to the change of focal point the $\Delta T$ dropped to approximately $80 \mathrm{~K}$ at the $V_{o c}$ of $2.5 \mathrm{~V}$. Fig. $5 \mathrm{~b}$ shows the maximum $I_{s c}$ of $0.75 \mathrm{~A}$ at the $\Delta T$ of $110 \mathrm{~K}$. Fig. $5 c$ shows the relationship between $\Delta T$ and output power and it can be seen that the maximum out power achieved was $2.7 \mathrm{~W}$ in the current setup and improved compared to the preceding experimental results. The temperature dependent figure of merit ZT also behaves similarly to the short-circuit current measurements and confirmed with theoretical calculations of ZT as shown in Fig. $5 \mathrm{~d}$. Fig. $5 \mathrm{e}$ and Fig. $5 \mathrm{f}$ shows the $V_{o c}$ and $I_{s c}$ relationship against the average temperature $T_{a v}$, respectively. It can be seen that the $V_{o c}$ and $I_{s c}$ increases somehow linearly with respect to the change in $T_{a v}$. 

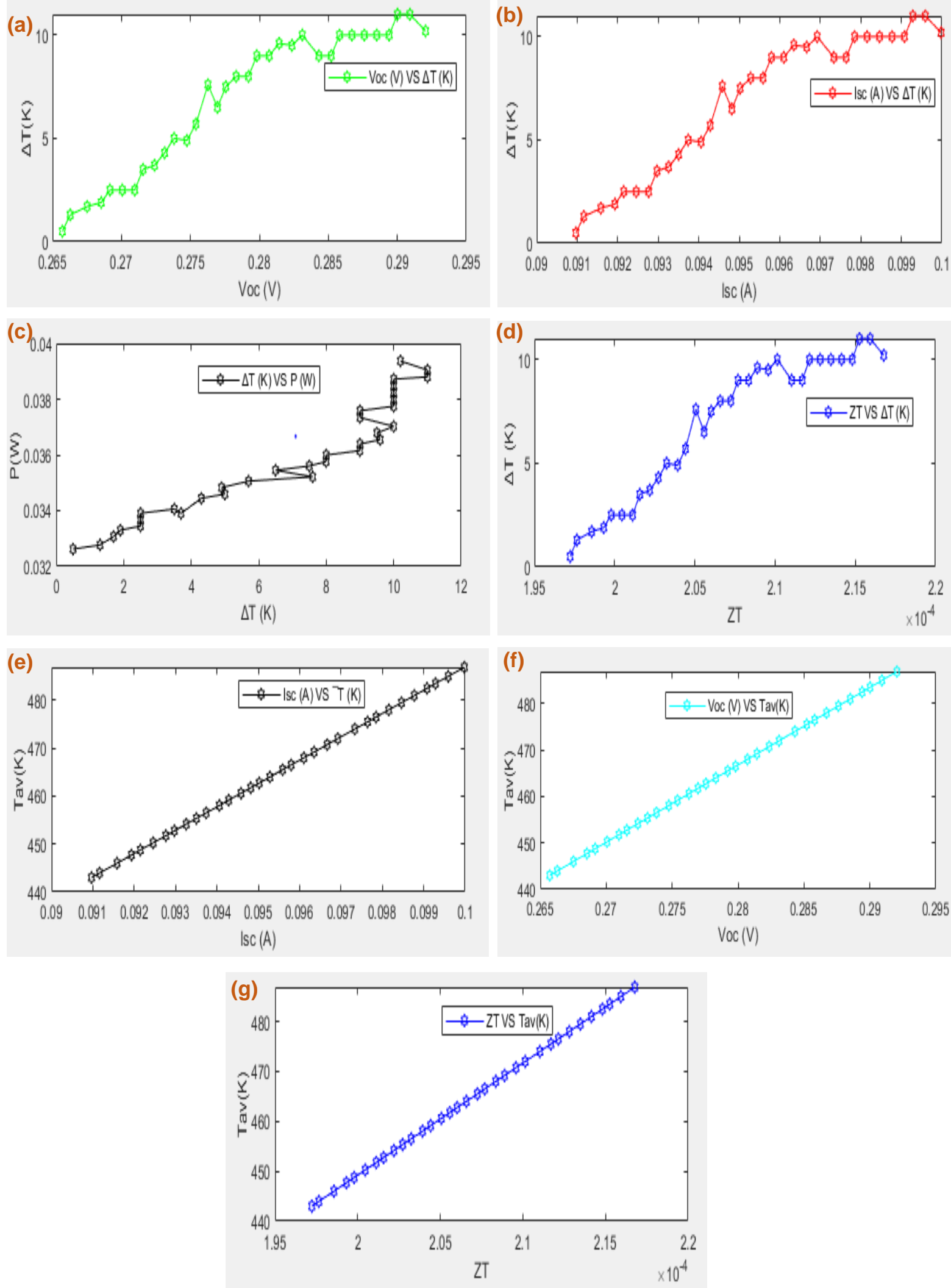

Fig. 4. Experimental results of the TEG-setup with quartz-halogen concentrated heat showing (a) $V_{o c}$ versus $\Delta T$, (b) $I_{s c}$ versus $\Delta T$, (c) $P$ versus $V_{o c}$ versus $\Delta T$, (d) $Z T$ versus $\Delta T$, (e) $T_{a v}$ versus $I_{s c}$, (f) $T_{a v}$ versus $V_{o c}$, and (g) $T_{a v}$ versus $Z T$ 

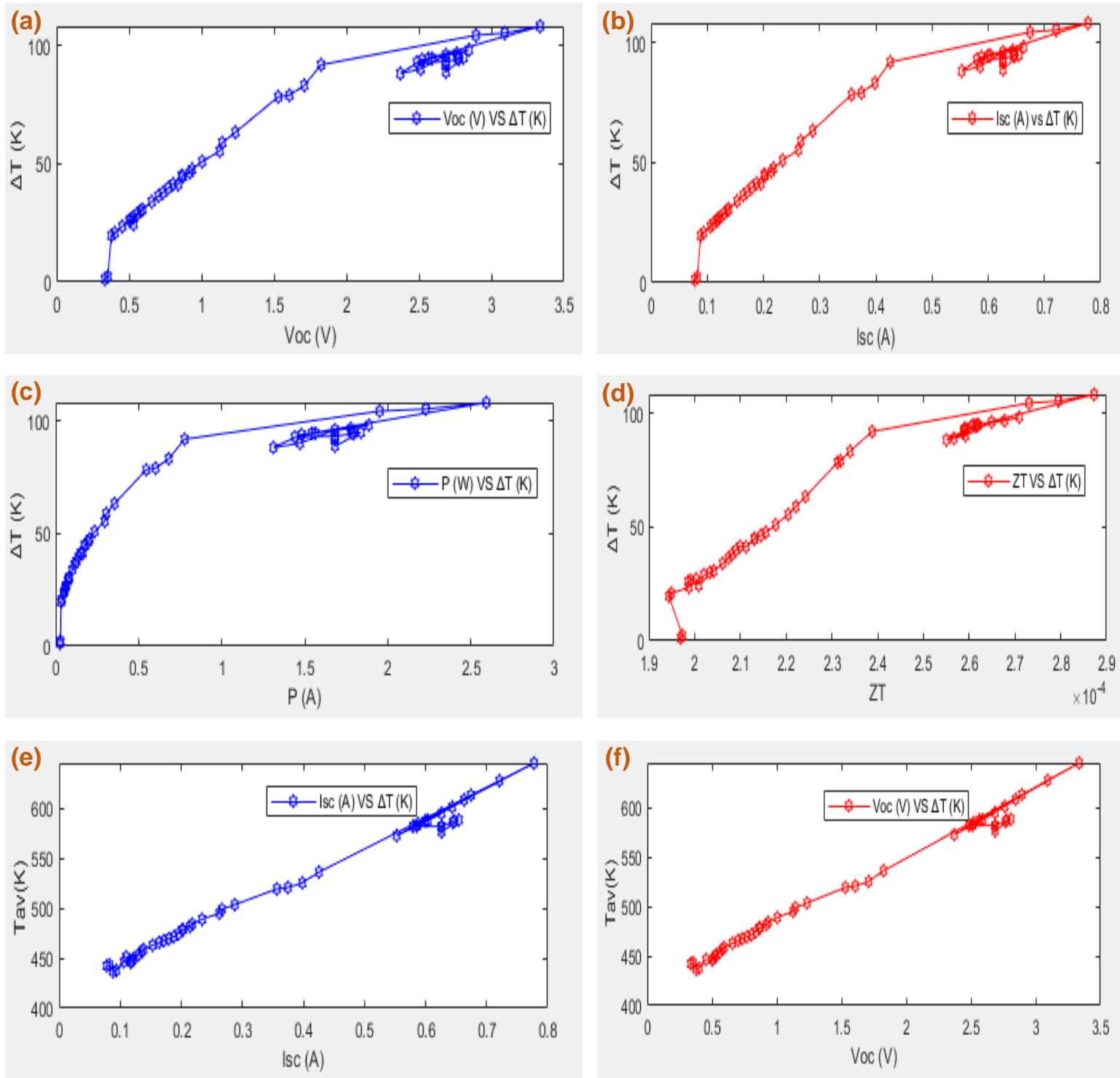

Fig. 5. Experimental results of the TEG-setup with natural solar thermal concentrated heat harvesting showing (a) $V_{o c}$ versus $\Delta T$, (b) $I_{s c}$ versus $\Delta T$, (c) $\Delta T$ versus $P$, (d) $\Delta T$ versus $Z T$, (e) $I_{s c}$ versus $T_{a v}$ and, (f) $T_{a v}$ versus $V_{o c}$

\section{Conclusions}

This research study presented the experimental and theoretical analysis on the electrical performances of TEG in harnessing and concentrating the natural sunlight and quartz-halogen heat utilizing the parabolic trough mirror. The experiment is conducted by utilizing several heat sources expecting a difference in the output of the TEG. These experiments utilize parabolic trough mirror for conversion of solar radiations, which are emitted from the source to the mirror to concentrate it on the hot side of TEG. Several experiments were conducted and reported here to that are: TEG-setup with eight quartz-halogen concentrated heat and natural solar heat concentration. The experimental results are validated with the mathematical calculations. The Parabolic trough mirror used has a focal point at a distance of $19.05 \mathrm{~cm}$. With eight quartz-halogen concentrated heat, maximum performance achieved at $\Delta T$ of $11.8 \mathrm{~K}, V_{o c}$ of $292 \mathrm{mV}$ and $I_{s c}$ of $95.8 \mathrm{~mA}$, recorded at the concentrated hot-side surface temperature of $317.8 \mathrm{~K}$. When compared to the natural solar concentrated heat, higher temperature of $473.15 \mathrm{~K}$ at the hot-side surface temperature of TG was achieved. It is 
concluded that the heat concentration of the parabolic mirror increases with an increase of the intensity of heat using natural solar radiations. The $V_{o c}$ of $1.76 \mathrm{~V}$ and $I_{s c}$ of $1.1 \mathrm{~A}$ at a temperature difference of $110 \mathrm{~K}$ were measured, which are in good agreement with mathematical results. The parabolic through mirror utilized is a small in size and thus collected lesser sunrays than the larger dish style mirror and hence the heat in the focal point was very low, for better results, parabolic trough mirror with higher surface area would be important for future experiments.

\section{Acknowledgement}

This research work was supported by the international collaboration with Hokkaido University, Yangzhou University, Benha University and London South Bank University Solar Thermal Vacuum Engineering Research Group. This research work was not funded by any grant and is a self-initiative collaborative research work.

\section{References}

[1] Memon, Saim. "Advanced Thermoelectric Materials for Energy Harvesting Applications." (2020). IntechOpen Publisher, London, ISBN: 978-1-78984-529-7 https://doi.org/10.5772/intechopen.77430.

[2] Memon, Saim. "Introductory Chapter: Introduction to Advanced Thermoelectric Materials for Energy Harvesting Applications." 2019. https://doi.org/10.5772/intechopen.89640.

[3] Wang, Qiang, Xianxian Liu, and Xiuli Guo. "Application of waste heat recovery technology in union station and analysis of energy efficiency." Procedia Engineering 205 (2017): 3860-3866. https://doi.org/10.1016/j.proeng.2017.10.063

[4] Wu, Yongjia, Lei Zuo, Jie Chen, and Jackson A. Klein. "A model to analyze the device level performance of thermoelectric generator." Energy 115 (2016): 591-603.

https://doi.org/10.1016/j.energy.2016.09.044

[5] Goldsmid, H. J., and R. W. Douglas. "The use of semiconductors in thermoelectric refrigeration." British Journal of Applied Physics 5, no. 11 (1954): 386. https://doi.org/10.1088/0508-3443/5/11/303

[6] Chen, Wei-Hsin, Chien-Chang Wang, Chen-I. Hung, Chang-Chung Yang, and Rei-Cheng Juang. "Modeling and simulation for the design of thermal-concentrated solar thermoelectric generator." Energy 64 (2014): 287-297. https://doi.org/10.1016/j.energy.2013.10.073

[7] Saravanan, Ramachandran. "Charge Density and Structural Characterization of Thermoelectric Materials." Materials Research Forum LLC, 2016. ISBN: 9781945291012.

[8] Choi, Hyeongdo, Yong Jun Kim, Choong Sun Kim, Hyeong Man Yang, Min-Wook Oh, and Byung Jin Cho. "Enhancement of reproducibility and reliability in a high-performance flexible thermoelectric generator using screen-printed materials." Nano Energy 46 (2018): 39-44.

https://doi.org/10.1016/j.nanoen.2018.01.031

[9] Pineda, Diana Davila, Oliver Brand, Gary K. Fedder, Christofer Hierold, Jan G. Korvink, and Osamu Tabata. "Thermoelectric energy conversion: basic concepts and device applications". John Wiley \& Sons, Incorporated, (2017).

[10] Kraemer, Daniel, Bed Poudel, Hsien-Ping Feng, J. Christopher Caylor, Bo Yu, Xiao Yan, Yi Ma et al. "Highperformance flat-panel solar thermoelectric generators with high thermal concentration." Nature materials 10, no. 7 (2011): 532-538.

https://doi.org/10.1038/nmat3013

[11] Memon, Saim, and Khawaja Noman Tahir. "Experimental and analytical simulation analyses on the electrical performance of thermoelectric generator modules for direct and concentrated quartz-halogen heat harvesting." Energies 11, no. 12 (2018): 3315. https://doi.org/10.3390/en11123315.

[12] He, Hailong, Yi Wu, Weiwei Liu, Mingzhe Rong, Zhenxuan Fang, and Xiaojun Tang. "Comprehensive modeling for geometric optimization of a thermoelectric generator module." Energy conversion and management 183 (2019): 645-659.

https://doi.org/10.1016/j.enconman.2018.12.087 
[13] Habibollahzade, Ali, Ehsan Gholamian, Pouria Ahmadi, and Amirmohammad Behzadi. "Multi-criteria optimization of an integrated energy system with thermoelectric generator, parabolic trough solar collector and electrolysis for hydrogen production." International journal of hydrogen energy 43, no. 31 (2018): 14140-14157.

https://doi.org/10.1016/j.ijhydene.2018.05.143

[14] Alam, Hilaal, and Seeram Ramakrishna. "A review on the enhancement of figure of merit from bulk to nanothermoelectric materials." Nano energy 2, no. 2 (2013): 190-212. https://doi.org/10.1016/j.nanoen.2012.10.005

[15] Zhao, Li-Dong, Bo-Ping Zhang, Jing-Feng Li, Min Zhou, Wei-Shu Liu, and Jing Liu. "Thermoelectric and mechanical properties of nano-SiC-dispersed Bi2Te3 fabricated by mechanical alloying and spark plasma sintering." Journal of Alloys and Compounds 455, no. 1-2 (2008): 259-264. https://doi.org/10.1016/j.jallcom.2007.01.015

[16] Rodríguez, J. E., D. Cadavid, and L. C. Moreno. "Thermoelectric figure of merit of LSCoO-Mn perovskites." Microelectronics journal 39, no. 11 (2008): 1236-1238. https://doi.org/10.1016/j.mejo.2008.01.046

[17] Boyer, A., and E. Cisse. "Properties of thin film thermoelectric materials: application to sensors using the Seebeck effect." Materials Science and Engineering: B 13, no. 2 (1992): 103-111. https://doi.org/10.1016/0921-5107(92)90149-4

[18] Mohsenzadeh, Milad, and M. B. Shafii. "A novel concentrating photovoltaic/thermal solar system combined with thermoelectric module in an integrated design." Renewable Energy 113 (2017): 822-834. https://doi.org/10.1016/j.renene.2017.06.047

[19] Liu, ZhongBing, Ling Zhang, GuangCai Gong, HangXin Li, and GuangFa Tang. "Review of solar thermoelectric cooling technologies for use in zero energy buildings." Energy and Buildings 102 (2015): 207-216. https://doi.org/10.1016/j.enbuild.2015.05.029

[20] Nemir, David, and Jan Beck. "On the significance of the thermoelectric figure of merit Z." Journal of electronic materials 39, no. 9 (2010): 1897-1901. https://doi.org/10.1007/s11664-009-1060-4

[21] Al-Azzawi, Abdul. Physical optics: principles and practices. CRC Press, 2018.

[22] Tsai, Huan-Liang, and Jium-Ming Lin. "Model building and simulation of thermoelectric module using Matlab/Simulink." Journal of Electronic Materials 39, no. 9 (2010): 2105. https://doi.org/10.1007/s11664-009-0994-x.

[23] Elarusi, Abdulmunaem H., Hassan Fagehi, Hosung Lee, and Alaa Attar. "Theoretical approach to predict the performance of thermoelectric generator modules." Journal of Electronic Materials 46, no. 2 (2017): 872-881. https://doi.org/10.1007/s11664-016-4948-9

[24] Goldsmid, H. Julian. Introduction to thermoelectricity. Vol. 121. Berlin: Springer, 2010.

[25] Goldsmid, H. Julian. "Bismuth telluride and its alloys as materials for thermoelectric generation." Materials 7, no. 4 (2014): 2577-2592. https://doi.org/10.3390/ma7042577

[26] Kane, Aarti, Vishal Verma, and Bhim Singh. "Temperature dependent analysis of thermoelectric module using Matlab/SIMULINK." In 2012 IEEE International Conference on Power and Energy (PECon), pp. 632-637. IEEE, 2012. https://doi.org/ 10.11648/j.ijepe.20160503.12.

[27] Kobbekaduwa, K. P. V. B., and N. D. Subasinghe. "Modelling and Analysis of Thermoelectric Generation of Materials Using Matlab/Simulink." International Journal of Energy and Power Engineering 5, no. 3 (2016): 97. https://doi.org/10.11648/j.ijepe.20160503.12

[28] Kıyan, Metin, Ekin Bingöl, Mehmet Melikoğlu, and Ayhan Albostan. "Modelling and simulation of a hybrid solar heating system for greenhouse applications using Matlab/Simulink." Energy Conversion and Management 72 (2013): 147-155. https://doi.org/10.1016/j.enconman.2012.09.036

[29] Khattab, N. M., and E. T. El Shenawy. "Optimal operation of thermoelectric cooler driven by solar thermoelectric generator." Energy Conversion and Management 47, no. 4 (2006): 407-426. https://doi.org/10.1016/j.enconman.2005.04.011

[30] He, Hailong, Yi Wu, Weiwei Liu, Mingzhe Rong, Zhenxuan Fang, and Xiaojun Tang. "Comprehensive modeling for geometric optimization of a thermoelectric generator module." Energy conversion and management 183 (2019): 645-659. https://doi.org/10.1016/j.enconman.2018.12.087

[31] Köysal, Yavuz. "Performance analysis on solar concentrating thermoelectric generator coupled with heat sink." International Journal of Precision Engineering and Manufacturing 20, no. 2 (2019): 313-318. https://doi.org/10.1007/s12541-019-00060-w 
[32] Özdemir, Ali Ekber, Yavuz Köysal, Engin Özbaş, and Tahsin Atalay. "The experimental design of solar heating thermoelectric generator with wind cooling chimney." Energy Conversion and Management 98 (2015): 127-133. https://doi.org/10.1016/j.enconman.2015.03.108 